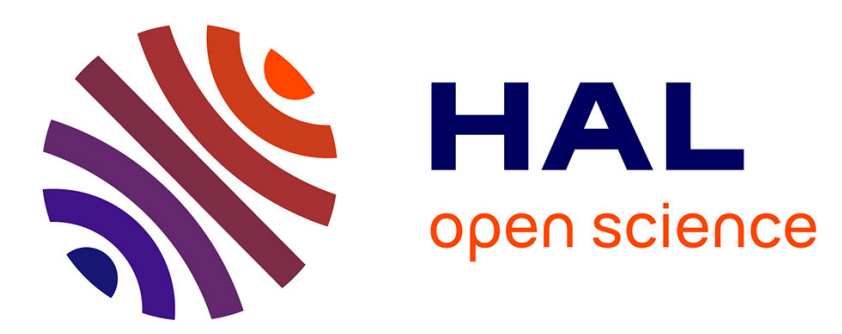

\title{
Monotone combined finite volume-finite element scheme for a bone healing model
}

Marianne Bessemoulin-Chatard, Mazen Saad

\section{To cite this version:}

Marianne Bessemoulin-Chatard, Mazen Saad. Monotone combined finite volume-finite element scheme for a bone healing model. Finite Volumes for Complex Applications VII-Elliptic, Parabolic and Hyperbolic Problems, Jun 2014, Berlin, Germany. pp.497-505, 10.1007/978-3-319-05591-6_49 . hal01015935

\section{HAL Id: hal-01015935 \\ https://hal.science/hal-01015935}

Submitted on 27 Jun 2014

HAL is a multi-disciplinary open access archive for the deposit and dissemination of scientific research documents, whether they are published or not. The documents may come from teaching and research institutions in France or abroad, or from public or private research centers.
L'archive ouverte pluridisciplinaire HAL, est destinée au dépôt et à la diffusion de documents scientifiques de niveau recherche, publiés ou non, émanant des établissements d'enseignement et de recherche français ou étrangers, des laboratoires publics ou privés. 


\title{
Monotone combined finite volume-finite element scheme for a bone healing model
}

\author{
Marianne Bessemoulin-Chatard and Mazen Saad
}

\begin{abstract}
We define a combined edge FV-FE scheme for a bone healing model. This choice of discretization allows to take into account anisotropic diffusions and does not impose any restrictions on the mesh. Moreover, following [3], we propose a nonlinear correction to obtain a monotone scheme. We present some numerical experiments which show its good behavior.
\end{abstract}

\section{Introduction}

We consider a bone growth model based on [1]. It describes the evolution of the concentrations of the following quantities: the mesenchymal stem cells $s$, the osteoblasts $b$, the bone matrix $m$ and the osteogenic growth factor $g$. Bone healing begins by the migration of the stem cells to the site of the injury. Then along the bone, these cells differentiate into osteoblasts which start to synthetize the bone matrix. This cell differentiation is only possible in presence of the growth factor.

The proposed model takes into account several phenomena: the diffusion of the stem cells and the growth factor, the migration of the stem cells towards the bone matrix, the proliferation and the differentiation of stem cells. The osteoblasts are considered without movement since they are fixed at the bone matrix. Moreover, the model includes the case of heterogeneous domains, with possibly anisotropic diffusions. It is given by the following nonlinear coupled system: for $t>0$ and $x \in \Omega$, where $\Omega$ is an open bounded polyhedral subset of $\mathbb{R}^{d}, d=2,3$,

M. Bessemoulin-Chatard

Université de Nantes, LMJL - UMR6629, 2 rue de la Houssinière - BP 9220844322 Nantes Cedex

3, France e-mail: Marianne.Bessemoulin@univ-nantes.fr

M. Saad

Ecole Centrale de Nantes, LMJL - UMR6629,1, rue de la Noé, BP 92101, 44321 Nantes Cedex 3, France e-mail: Mazen.Saad@ec-nantes.fr 


$$
\begin{gathered}
\partial_{t} s-\operatorname{div}(\mathbf{S}(x)(\Lambda(m) \nabla s-V(m) \chi(s) \nabla m))=K_{1}(m) \chi(s)-H(g) s \\
\partial_{t} b=K_{2}(m) \chi(b)+\rho H(g) s-\delta_{1} b \\
\partial_{t} m=\lambda(1-m) b \\
\partial_{t} g-\operatorname{div}\left(\mathbf{S}(x) \Lambda_{g} \nabla g\right)=P(g) b-\delta_{2} g .
\end{gathered}
$$

The functions $K_{1}(m), K_{2}(m), H(g), P(m)$ and the positive parameters $\rho, \delta_{1}, \lambda$ and $\delta_{2}$ are given (see [1]). The diffusion coefficient $\Lambda(m)$ and the haptotaxis velocity $V(m)$ are given by

$$
\Lambda(m)=\frac{\chi_{h}}{\zeta_{h}^{2}+m^{2}}\left(m+\Lambda_{0}\right)(1-m), \quad V(m)=\frac{\chi_{k}}{\left(\zeta_{k}+m\right)^{2}},
$$

with $\chi_{h}, \zeta_{h}, \Lambda_{0}, \chi_{k}, \zeta_{k}>0$. The diffusion coefficient $\Lambda_{g}$ for the growth factor is a positive constant. Moreover, the accumulation of stem cells is limited by the factor $\chi(s)=s(1-s)$. The permeability $\mathbf{S}(x)$ is a symmetric $d \times d$ matrix, with $\mathbf{S} \in L^{\infty}(\Omega)$, and we assume that $\exists C_{S}>0$ such that $\forall x \in \Omega, \forall \xi \in \mathbb{R}^{d}, \mathbf{S}(x) \xi \cdot \xi \geq C_{S}|\xi|^{2}$.

This nonlinear system (1)-(4) is supplemented with initial conditions $s_{0}, b_{0}, m_{0}, g_{0}$ and with homogeneous Neumann boundary conditions on $s$ and $g$ :

$$
\mathbf{S}(x)\left(\Lambda_{1}(m) \nabla s-V(m) \chi(s) \nabla m\right) \cdot \mathbf{n}=0, \quad \mathbf{S}(x) \Lambda_{g} \nabla g \cdot \mathbf{n}=0,
$$

for $t \in(0, T)$ and $x \in \partial \Omega$, where $\mathbf{n}$ is the outward unit normal of $\partial \Omega$. Following [5], a solution $u=(s, b, m, g)$ is said to be physically admissible if $u \in \mathscr{A}=$ $[0,1] \times[0, \bar{b}] \times[0,1] \times[0, \bar{g}]$, where $\bar{b}$ and $\bar{g}$ depend on the physical parameters.

In this paper, we propose a numerical scheme for this bone growth model. A finite volume (FV) scheme was proposed in [5] for this model in homogeneous domains where the diffusion tensor $\mathbf{S}=I d$. The cell-centered FV method with an upwind discretization of the convective terms provides the stability and is extremely robust. However in this case, the mesh is assumed to be admissible [7, Definition 9.1]. In particular, this implies that the orthogonality condition has to be satisfied. As mentioned in [5], a difficulty in the implementation is to construct such admissible meshes. Structured rectangular meshes are admissible, but they cannot be used for complex geometries arising in physical contexts. Furthermore, the finite element (FE) method allows for an easy discretization of diffusive terms with full tensors without imposing any restrictions on the meshes. However, some numerical instabilities may arise in the convection-dominated case.

The idea is hence to combine a FE discretization of diffusive terms with a FV discretization of the other terms. Such schemes were proposed and studied in [9] for fluid mechanics equations in the case of diffusion terms with $\mathbf{S}=I d$ and in [4] for anisotropic Keller-Segel model. This idea was extended in [8] to inhomogeneous and anisotropic diffusion-dispersion tensors and to very general meshes only satisfying the shape regularity condition (6). However, the maximum principle is no more guaranteed if there exist negative transmissibilities. 
We first introduce in Section 2 the combined FV-FE scheme for the bone healing model (1)-(4). Then in Section 3 we apply the method described in [3] to construct a nonlinear correction providing a discrete maximum principle. Finally in Section 4 we present some numerical experiments showing the efficiency of the scheme.

\section{The combined FV-FE scheme}

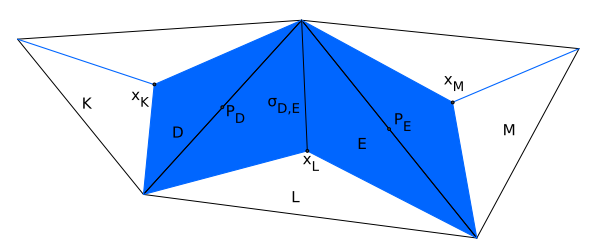

Fig. 1 Triangles $K, L$ and $M \in \mathscr{T}$ and diamonds $D, E \in \mathscr{D}$ associated with edges $\sigma_{D}, \sigma_{E} \in \mathscr{E}$.

A mesh of $\Omega$ is a family $\mathscr{T}$ of closed simplices $K$ such that $\bar{\Omega}=\cup_{K \in \mathscr{T}} K$. We denote by $\mathscr{E}$ the set of all edges, by $\mathscr{E}^{\text {int }}$ the set of interior edges, by $\mathscr{E}$ ext the set of boundary edges and by $\mathscr{E}_{K}$ the set of all edges of $K \in \mathscr{T}$. The size of the mesh is defined by $h:=\max \operatorname{diam}(K)$. We assume that there exists a positive constant $k_{\mathscr{T}}$ such that:

$$
\operatorname{reg}(\mathscr{T}):=\min _{K \in \mathscr{T}} \frac{|K|}{(\operatorname{diam}(K))^{d}} \geq k_{\mathscr{T}} .
$$

We also use a dual partition $\mathscr{D}$ of control volumes $D$ of $\Omega$ called diamonds such that $\bar{\Omega}=\cup_{D \in \mathscr{D}} D$. Each diamond $D$ is associated with one edge $\sigma_{D} \in \mathscr{E}$. We construct it by connecting the barycenters of every $K \in \mathscr{T}$ that contains $\sigma_{D}$ through the vertices of $\sigma_{D}$. For $\sigma_{D} \in \mathscr{E}$ ext , the contour of $D$ is completed by the edge $\sigma_{D}$ itself. We define $\mathscr{D}^{\text {int }}$ and $\mathscr{D}^{\text {ext }}$ the set of all interior and boundary dual volumes respectively. For $K \in \mathscr{T}$, we set $\mathscr{D}_{K}:=\left\{D \in \mathscr{D} ; \sigma_{D} \in \mathscr{E}_{K}\right\}$. We denote by $|D|$ the $d$-dimensional Lebesgue measure of $D$ and $|\sigma|$ the $(d-1)$-dimensional measure of $\sigma$. For all $D \in \mathscr{D}, P_{D}$ is the barycenter of $\sigma_{D}$ and $\mathscr{N}(D)$ is the set of neighbours of $D$. For all $D \in \mathscr{D}$ and all $E \in \mathscr{N}(D), \sigma_{D, E}$ is the interface between $D$ and $E$ and $\mathbf{n}_{D, E}$ is the unit normal vector to $\sigma_{D, E}$ outward to $D$.

Next we define the following finite-dimensional space of piecewise linear nonconforming $\mathrm{FE}[6]$ :

$X:=\left\{\varphi \in L^{2}(\Omega) ;\left.\varphi\right|_{K}\right.$ linear $\forall K \in \mathscr{T}, \varphi$ continuous at the points $\left.P_{D}, D \in \mathscr{D}^{\text {int }}\right\}$, equipped with the seminorm $\|u\|_{X}^{2}:=\sum_{K \in \mathscr{T}} \int_{K}|\nabla u|^{2} d x$.

The basis of $X$ is spanned by the shape functions $\varphi_{D}, D \in \mathscr{D}$, such that $\varphi_{D}\left(P_{E}\right)=$ $\delta_{D E}, E \in \mathscr{D}$. The approximations in this space are nonconforming since $X \nsubseteq H^{1}(\Omega)$. 
Finally, we define the time step $\Delta t$ and the increasing sequence $\left(t^{n}\right)_{0 \leq n \leq N+1}$, where $t^{n}=n \Delta t$ and $N$ is the smallest integer such that $(N+1) \Delta t \geq T$.

The discrete unknowns are denoted by $\left\{w_{D}^{n}, D \in \mathscr{D}, n \in\{0 \cdots N+1\}\right\}$, where the value $w_{D}^{n}$ is an approximation of $w\left(P_{D}, t^{n}\right), w=s, b, m, g$.

We now define the semi-implicit in time and combined FV-FE in space discretization for (1)-(4). The initial conditions are approximated by $\left(s_{D}^{0}, b_{D}^{0}, m_{D}^{0}, g_{D}^{0}\right)_{D \in \mathscr{D}}$ by taking the mean values of $s_{0}, b_{0}, m_{0}$ and $g_{0}$ on each dual cell $D$. Then the scheme is given by the following set of equations: for all $n \in\{0, \ldots, N\}$ and all $D \in \mathscr{D}^{\text {int }}$,

$$
\begin{aligned}
& |D|\left(s_{D}^{n+1}-s_{D}^{n}\right)-\Delta t \sum_{E \in \mathscr{D}^{\text {int }}} \Lambda_{D, E}^{n} s_{E}^{n+1}+\Delta t \sum_{E \in \mathscr{N}(D)} G\left(s_{D}^{n+1}, s_{E}^{n+1}, V_{D, E}^{n}\left(m_{E}^{n+1}-m_{D}^{n+1}\right)\right) \\
& \quad=\Delta t|D|\left(K_{1}\left(m_{D}^{n}\right) s_{D}^{n+1}\left(1-s_{D}^{n}\right)-H\left(g_{D}^{n}\right) s_{D}^{n+1}\right) \\
& |D|\left(b_{D}^{n+1}-b_{D}^{n}\right)=\Delta t|D|\left(K_{2}\left(m_{D}^{n}\right) \chi\left(b_{D}^{n+1}\right)+H\left(g_{D}^{n}\right) s_{D}^{n+1}-\delta_{1} b_{D}^{n+1}\right), \\
& |D|\left(m_{D}^{n+1}-m_{D}^{n}\right)=\Delta t|D| \lambda\left(1-m_{D}^{n+1}\right) b_{D}^{n+1} \\
& |D|\left(g_{D}^{n+1}-g_{D}^{n}\right)-\Delta t \sum_{E \in \mathscr{D}^{i n t}} \mathscr{S}_{D, E} \Lambda_{g} g_{E}^{n+1}=\Delta t|D|\left(P\left(g_{D}^{n}\right) b_{D}^{n}-\delta_{2} g_{D}^{n+1}\right)
\end{aligned}
$$

where for $U=\Lambda, V$,

$$
U_{D, E}=-\sum_{K \in \mathscr{T}} U_{K}\left(\mathbf{S}(x) \nabla \varphi_{E}, \nabla \varphi_{D}\right)_{0, K}, \quad \mathscr{S}_{D, E}=-\sum_{K \in \mathscr{T}}\left(\mathbf{S}(x) \nabla \varphi_{E}, \nabla \varphi_{D}\right)_{0, K},
$$

with $U_{K}=\frac{\sum_{D \in \mathscr{D}_{K}} U\left(m_{D}\right)}{\operatorname{card}\left(\mathscr{E}_{K}\right)}$.

The flux function $G$ is supposed to be monotone, consistent, conservative and locally Lipschitz continuous. For example, we consider in the following

$$
G(a, b, c)=c^{+}\left(\chi_{\uparrow}(a)+\chi_{\downarrow}(b)\right)-c^{-}\left(\chi_{\uparrow}(b)+\chi_{\downarrow}(a)\right),
$$

where $c^{+}=\max (c, 0), c^{-}=\max (-c, 0), \chi_{\uparrow}$ and $\chi_{\downarrow}$ are respectively the nondecreasing and nonincreasing parts of $\chi$.

Definition 1 (Approximate solution). Using the values $\left(u_{D}\right)_{D \in \mathscr{D}}, u=s, b, m, g$, we define a nonconforming FE solution $u_{h}$ as a function piecewise linear and continuous in the barycenters $P_{D}$ of interior edges such that

$$
u_{h}(x)=\sum_{D \in \mathscr{D}} u_{D} \varphi_{D}(x), \quad x \in \Omega .
$$

Properties of the discrete diffusive operators. We define

$$
\begin{array}{rlrl}
\mathscr{A}^{\mathscr{D}}: \mathbb{R}^{\operatorname{Card}(\mathscr{D})} \quad \rightarrow \mathbb{R}^{\operatorname{Card}(\mathscr{D})} & \mathscr{L}^{\mathscr{D}}: \mathbb{R}^{\operatorname{Card}(\mathscr{D})} & \rightarrow \mathbb{R}^{\operatorname{Card}(\mathscr{D})} \\
s_{h}=\left(s_{D}\right)_{D \in \mathscr{D}} & \mapsto\left(A_{D}\left(s_{h}\right)\right)_{D \in \mathscr{D}}, & g_{h}=\left(g_{D}\right)_{D \in \mathscr{D}} & \mapsto\left(L_{D}\left(g_{h}\right)\right)_{D \in \mathscr{D}}
\end{array},
$$

the discrete diffusive operators appearing in (7), (10), with for all $D \in \mathscr{D}$,

$$
A_{D}\left(s_{h}\right)=\sum_{E \in \mathscr{D}^{\text {int }}} \Lambda_{D, E} s_{E}, \quad L_{D}\left(g_{h}\right)=\sum_{E \in \mathscr{D}^{\text {int }}} \Lambda_{g} \mathscr{S}_{D, E} g_{E} .
$$


We give in the following proposition some properties of $\mathscr{A}^{\mathscr{D}}$ which are crucial to get the convergence of the scheme. The same results hold for $\mathscr{L}^{\mathscr{D}}$ too.

Proposition 1. The discrete diffusive operator $\mathscr{A}^{\mathscr{D}}$ is

- conservative: $\forall D \in \mathscr{D}, \quad A_{D}\left(s_{h}\right)=\sum_{E \in \mathscr{N}(D)} \Lambda_{D, E}\left(s_{E}-s_{D}\right)$,

- coercive: $\exists C_{A}>0$ such that $-\sum_{D \in \mathscr{D}} A_{D}\left(s_{h}\right) s_{D} \geq C_{A}\left\|s_{h}\right\|_{X}^{2} \quad \forall s_{h} \in X$.

\section{Monotone correction}

At this stage, the constructed scheme is valid both for full anisotropic diffusion tensors and for general meshes satisfying only assumption (6). However, it possesses a discrete maximum principle only if all transmissibilities $\Lambda_{D, E}, \mathscr{S}_{D, E}$ are nonnegative, which is not guaranteed in the general case. Following [3], we now define a nonlinear correction which gives monotone scheme while preserving the properties described in Proposition 1.

We replace the operator $\mathscr{A}^{\mathscr{D}}$ in (7) by the corrected operator $\mathscr{B}^{\mathscr{D}}$ defined by

$$
B_{D}(s)=A_{D}(s)+\sum_{E \in \mathscr{N}(D)} \beta_{D, E}^{\varepsilon}(s)\left(s_{D}-s_{E}\right) \quad \forall D \in \mathscr{D},
$$

where $\beta_{D, E}(s)$ is the regularized correction proposed in [3]:

$$
\beta_{D, E}^{\varepsilon}(s)=\max \left(\frac{\left|A_{D}(s)\right|}{\operatorname{Card}_{\varepsilon} V(D, s)^{*}}, \frac{\left|A_{E}(s)\right|}{\operatorname{Card}_{\varepsilon} V(E, s)^{*}}\right) \frac{1}{\left|s_{D}-s_{E}\right|+\varepsilon},
$$

with $\operatorname{Card}_{\varepsilon} V(D, s)^{*}=\sum_{E \in \mathscr{N}(D)} \frac{\left|s_{D}-s_{E}\right|}{\left|s_{D}-s_{E}\right|+\varepsilon}$.

This corrected diffusive operator is monotone since $\beta_{D, E}(s)>\left|A_{D}(s)\right|$ for all $D \in \mathscr{D}$, all $E \in \mathscr{N}(D)$. Moreover, the corrected diffusive operator $\mathscr{B}^{\mathscr{D}}$ still satisfies the properties described in Proposition 1:

- it is conservative, since $\beta_{D, E}=\beta_{E, D}$ for all $D \in \mathscr{D}, E \in \mathscr{N}(D)$,

- it is coercive, since $\beta_{D, E} \geq 0$ for all $D \in \mathscr{D}, E \in \mathscr{N}(D)$.

The diffusive operator $\mathscr{L}^{\mathscr{D}}$ can also be corrected in the same way.

Theorem 1. If $\left(s_{D}^{0}, b_{D}^{0}, m_{D}^{0}, g_{D}^{0}\right) \in \mathscr{A}$ for all $D \in \mathscr{D}$, then the discrete problem (7)(10) with monotone correction has a physically admissible solution $\left(s_{D}^{n}, b_{D}^{n}, m_{D}^{n}, g_{D}^{n}\right)$ $\in \mathscr{A}$, for all $n \geq 0$ and all $D \in \mathscr{D}$.

The proof of this result can be done by introducing a truncated version of the scheme (see [5, Theorem 5]), and using the properties of the corrected diffusive operators (monotony, conservativity, continuity). Following the same lines as [5, Theorem 7], we can also prove some energy estimates: 
Theorem 2. Let $\left(s_{D}^{n}, b_{D}^{n}, m_{D}^{n}, g_{D}^{n}\right)_{D \in \mathscr{D}, n \geq 0}$ be a solution of the corrected scheme. Then $\exists C>0$ not depending on the discretization parameters such that

$$
\sum_{n=0}^{N-1} \Delta t\left(\left\|s_{h}^{n}\right\|_{X}^{2}+\left\|b_{h}^{n}\right\|_{X}^{2}+\left\|m_{h}^{n}\right\|_{X}^{2}+\left\|g_{h}^{n}\right\|_{X}^{2}\right) \leq C .
$$

Starting from this result, one can obtain some compactness estimates on discrete solutions. The complete study of convergence of the corrected scheme, which requires some additional numerical assumptions [3], is done in [2].

\section{Numerical experiments}

We simulate the healing of a long bone fracture in rats [10]. The simulation corresponds to a $0.07 \mathrm{~cm}$ fracture. To implement the semi-implicit scheme (7)-(10), we use the Newton's method coupled with a biconjugate gradient method to solve the nonlinear system. While the discrete maximum principle is not satisfied, the monotone correction is computed using the iterative algorithm described in [3]. The geometry of the fracture and the initial condition are described on Fig. 2.

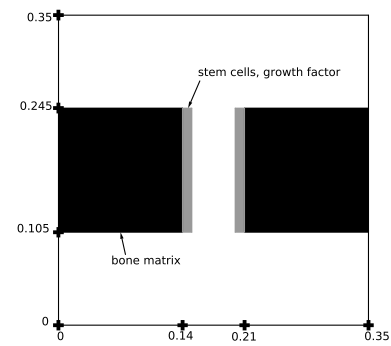

Fig. 2 Geometry and initial condition: the black area corresponds to the bone matrix $\left(m_{0}=1\right)$ and the grey area to the cellular cluster $\left(s_{0}=1, g_{0}=20\right)$. Elsewhere there is nothing initially.

We assume that $\mathbf{S}(x)=I_{2}$. We first consider an admissible mesh made of 14336 triangles and 21632 edges. Especially, all the angles are acute, which ensures in this case that the combined FV-FE scheme without correction satisfies the maximum principle. In particular, we observe that the discrete unknowns remain nonnegative (Tab. 1).

\begin{tabular}{|l|c|c|c|c|}
\hline & Min. Val. $s$ & Max. Val. $s$ & Min. Val. $g$ & Max. Val. g \\
\hline Iter. 1 & $9.47 \times 10^{-21}$ & 0.999 & $9.9 \times 10^{-21}$ & 19.8 \\
\hline Iter. 10 & $5.83 \times 10^{-21}$ & 0.991 & $9.05 \times 10^{-21}$ & 17.99 \\
\hline
\end{tabular}

Table 1 Results obtained with the non corrected scheme on an admissible mesh. 
Then we consider three general unstructured meshes that contain obtuse angles. Mesh 1 is made of 1539 triangles and 2346 edges, mesh 2 is made of 3132 triangles and 4756 edges, and mesh 3 is made of 15568 triangles and 23479 edges. In Tab. 2 , we present the minimum and maximum values obtained with the scheme before and after correction, after 1 and 10 iterations. We clearly observe that the discrete maximum principle is well respected after correction, with disappearance of the undershoots.

\begin{tabular}{|c|l|c|c|c|}
\hline & & Mesh 1 & Mesh 2 & Mesh 3 \\
\hline & Undershoots $s$ & 16 & 16 & 92 \\
& Min. Val. $s$ & $-2.67 \times 10^{-4}$ & $-8.95 \times 10^{-7}$ & $-3.01 \times 10^{-4}$ \\
Without & Max. Val. $s$ & 0.990 & 0.991 & 0.992 \\
correction & Undershoots $g$ & 70 & 71 & 144 \\
after 10 it. & Min. Val. $g$ & -0.27 & $-1.01 \times 10^{-2}$ & $-8.56 \times 10^{-3}$ \\
& Max. Val. $g$ & 17.96 & 18.41 & 20.27 \\
\hline With & Min. Val. $s$ & $9.58 \times 10^{-6}$ & $1.18 \times 10^{-6}$ & $3.93 \times 10^{-6}$ \\
correction & Max. Val. $s$ & 0.989 & 0.990 & 0.99 \\
after 10 it. & Min. Val. $g$ & $1.51 \times 10^{-4}$ & $8.02 \times 10^{-5}$ & $7.68 \times 10^{-5}$ \\
& Max. Val. $g$ & 17.81 & 18.33 & 19.37 \\
\hline
\end{tabular}

Table 2 Numerical results with the original and the corrected schemes after 10 iterations.

We now consider the corrected scheme on the finest mesh 3. After 2 days, we observe the formation of osteoblasts where the stem cells were initially concentrated (see Fig. 3). These osteoblasts synthetized the new bone matrix, which evolution is shown on Fig. 4. The stem cells moved towards the center of the fracture. These results are in agreement with previous results $[5,10]$.

Acknowledgements: M. B.-C. is supported by the ANR project GEONUM (ANR-12-IS01-0004-01).

\section{References}

1. Bailón-Plaza, A., Van Der Meulen, M.: A mathematical framework to study the effects of growth factor influences on fracture healing. J. Theor. Biol. 212(2) (2001)

2. Bessemoulin-Chatard, M., Saad, M.: Analysis of a monotone combined finite volume-finite element scheme for a bone healing model. In preparation

3. Cancès, C., Cathala, M., Le Potier, C.: Monotone coercive cell-centered finite volume schemes for anisotropic diffusion equations. Numer. Math. 125(3) (2013)

4. Chamoun, G., Saad, M., Talhouk, R.: Monotone combined edge finite volume-finite element scheme for anisotropic Keller-Segel model. Numer. Meth. Part. D. E. (2014)

5. Coudière, Y., Saad, M., Uzureau, A.: Analysis of a finite volume method for a bone growth system in vivo. Computers and Mathematics with Applications 66 (2013)

6. Crouzeix, M., Raviart, P.A.: Conforming and nonconforming finite element methods for solving the stationary Stokes equations. I. Rev. Française Automat. Informat. Recherche Opérationnelle Sér. Rouge 7 (1973)

7. Eymard, R., Gallouët, T., Herbin, R.: Finite volume methods. In: Handbook of numerical analysis, vol. VII. North-Holland, Amsterdam (2000) 


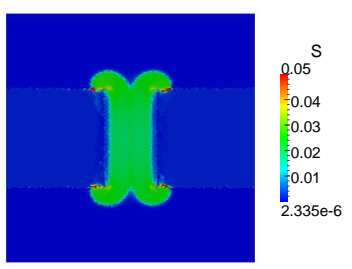

(a) Concentration of stem cells $s$

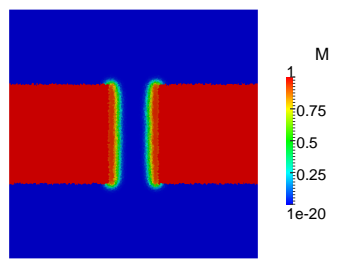

(c) Bone matrix density $m$

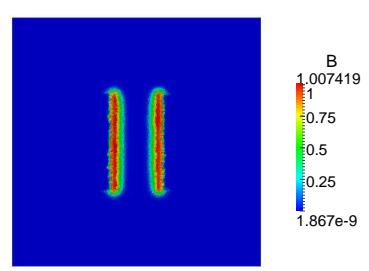

(b) Concentration of osteoblasts $b$

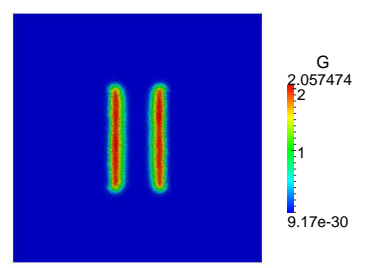

(d) Concentration of the growth factor $g$

Fig. 3 Bone matrix density, concentrations of stem cells, osteoblasts and growth factor at $T=2$ days.

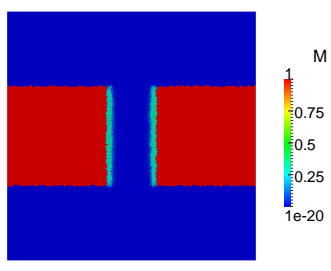

(a) $T=12$ hours

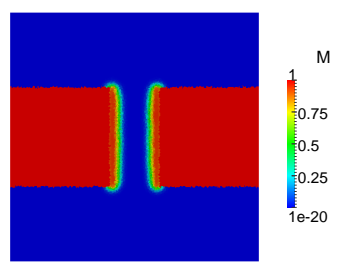

(b) $T=48$ hours

Fig. 4 Evolution of the bone matrix density.

8. Eymard, R., Hilhorst, D., Vohralik, M.: A combined finite volume-nonconforming/mixed hybrid finite element scheme for degenerate parabolic problems. Numer. Math. 105 (2006)

9. Feistauer, M., Felcman, J., Lukáčová-Medviďová, M.: Combined finite element-finite volume solution of compressible flow. J. Comput. Appl. Math. 63 (1995)

10. Uzureau, A.: Modélisations et calculs pour la cicatrisation osseuse. Application à la modélisation d'un bioréacteur. Ph.D. thesis, Université de Nantes (2012) 